\title{
Large scale analysis of protein stability in OMIM disease related human protein variants
}

Pier Luigi Martelli ${ }^{1,2^{*}+}$, Piero Fariselli ${ }^{1,3+}$, Castrense Savojardo ${ }^{1,2+}$, Giulia Babbi ${ }^{1,2}$, Francesco Aggazio ${ }^{1,2}$ and Rita Casadio ${ }^{1,2}$

From Varl-SIG at ISMB 2015

Dublin, Ireland. 11/07/2015

\begin{abstract}
Background: Modern genomic techniques allow to associate several Mendelian human diseases to single residue variations in different proteins. Molecular mechanisms explaining the relationship among genotype and phenotype are still under debate. Change of protein stability upon variation appears to assume a particular relevance in annotating whether a single residue substitution can or cannot be associated to a given disease. Thermodynamic properties of human proteins and of their disease related variants are lacking. In the present work, we take advantage of the available three dimensional structure of human proteins for predicting the role of disease related variations on the perturbation of protein stability.

Results: We develop INPS3D, a new predictor based on protein structure for computing the effect of single residue variations on protein stability $(\Delta \Delta \mathrm{G})$, scoring at the state-of-the-art (Pearson's correlation value of the regression is equal to 0.72 with mean standard error of $1.15 \mathrm{kcal} / \mathrm{mol}$ on a blind test set comprising 351 variations in 60 proteins). We then filter 368 OMIM disease related proteins known with atomic resolution (where the three dimensional structure covers at least $70 \%$ of the sequence) with 4717 disease related single residue variations and 685 polymorphisms without clinical consequence. We find that the effect on protein stability of disease related variations is larger than the effect of polymorphisms: in particular, by setting to $|1 \mathrm{kcal} / \mathrm{mol}|$ the threshold between perturbing and not perturbing variations of the protein stability, about $44 \%$ of disease related variations and $20 \%$ of polymorphisms are predicted with $|\Delta \Delta \mathrm{G}|>$ $1 \mathrm{kcal} / \mathrm{mol}$, respectively. A consistent fraction of OMIM disease related variations is however predicted to promote $|\Delta \Delta \mathrm{G}| \leq 1 \mathrm{kcal} / \mathrm{mol}$ and we focus here on detecting features that can be associated to the thermodynamic property of the protein variant. Our analysis reveals that some $47 \%$ of disease related variations promoting $|\Delta \Delta G| \leq 1$ are located in solvent exposed sites of the protein structure. We also find that the increase of the fraction of variations that in proteins are predicted with $|\Delta \Delta G| \leq 1 \mathrm{kcal} / \mathrm{mol}$, partially relates with the increasing number of the protein interacting partners, corroborating the notion that disease related, non-perturbing variations are likely to impair protein-protein interaction (70\% of the disease causing variations, with high accessible surface are indeed predicted in interacting sites). The set of OMIM surface accessible variations with $|\Delta \Delta \mathrm{G}| \leq 1 \mathrm{kcal} / \mathrm{mol}$ and located in interaction sites are $23 \%$ of the total in 161 proteins. Among these, 43 proteins with some 327 disease causing variations are involved in signalling, structural biological processes, development and differentiation.

(Continued on next page)
\end{abstract}

\footnotetext{
* Correspondence: gigi@biocomp.unibo.it

${ }^{\dagger}$ Equal contributors

'Biocomputing Group, University of Bologna, Via San Giacomo 9/2, 40126 Bologna, Italy

${ }^{2}$ Department BiGeA, University of Bologna, Via Selmi 3, 40126 Bologna, Italy

Full list of author information is available at the end of the article
} 
(Continued from previous page)

Conclusions: We compute the effect of disease causing variations on protein stability with INPS3D, a new state-of-the-art tool for predicting the change in $\Delta \Delta G$ value associated to single residue substitution in protein structures. The analysis indicates that OMIM disease related variations in proteins promote a much larger effect on protein stability than polymorphisms non-associated to diseases. Disease related variations with a slight effect on protein stability $(|\Delta \Delta \mathrm{G}|<1$ $\mathrm{kcal} / \mathrm{mol}$ ) frequently occur at the protein accessible surface suggesting that they are located in protein-protein interactions patches in putative human biological functional networks. The hypothesis is corroborated by proving that proteins with many disease related variations that slightly perturb protein stability are on average more connected in the human physical interactome (IntAct) than proteins with variations predicted with $|\Delta \Delta \mathrm{G}|>1 \mathrm{kcal} / \mathrm{mol}$.

Keywords: Protein stability, Disease related-variations, Residue solvent accessibility, Interactomics networks

\section{Background}

One of the key goals in the postgenomic era is the elucidation of the mechanisms at the basis of the relationship between genotype and phenotype. In particular, understanding how human genetic variations are associated to diseases is still an open problem and its solution is a crucial issue for exploiting the possibilities offered by the modern sequencing techniques in the framework of precision medicine $[1,2]$.

The role of missense mutations inducing single residue variations (SRVs) in proteins has been widely investigated: several databases collect data about the relationship between SRVs and diseases [3] and several predictive tools have been implemented in order to exploit the available knowledge to predict whether new variants are related to diseases ([4-6]; and others listed in [7]) or are affecting protein function [8].

Biophysical studies allowed to measure the thermodynamic effect that protein variations induce on protein stability [9]. However the number of human proteins whose folding thermodynamics is known in the native and mutated form is still limited due to the time consuming and costly procedure at the basis of experimental investigations. To fill the gap, predictive tools have been trained on the available thermodynamic data to compute the free energy change value upon variation ([10-13], and others listed in [14]). Recently, we introduced INPS [15], a sequence based predictor that well compares with tools taking as input protein structure. When dealing with disease related variations in human protein variants, very little is known about their thermodynamics and it is unclear in annotation processes whether a variation perturbing the protein stability is or not disease related. Extensive comparative analyses of the two classes of datasets (phenotypically vs thermodynamically characterized variations) prove that, on average, variation types most involved in disease are also associated to a large effect on protein stability [16-18]. However, the strength of this association, although recently improved (compare results in [16] with [19]), is not sufficient to consider protein destabilization as the only mechanistic cause explaining the insurgence of diseases. Indeed many variations with $|\Delta \Delta \mathrm{G}| \leq 1 \mathrm{kcal} /$ mol are disease-related $[12,13,15,16,19]$. In this paper, as a follow up to the problem, we specifically deal with OMIM disease related protein variants whose native structure is known and predict the extent of perturbation that the variation may cause on the native protein stability. To this aim, we develop INPS3D, a new tool for computationally estimating the effect of single residue variations on protein stability based on information extracted from protein three dimensional structure, and compare its performance to state-of-the-art predictors on the blind test set of the OMIM related proteins endowed with well resolved structures. By this, we identify a subset of disease-related variations with $|\Delta \Delta \mathrm{G}| \leq 1 \mathrm{kcal} / \mathrm{mol}$ and prove that these variations often occurs in sites exposed on the protein accessible surface, with a likelihood to be in interaction sites. Integrating these results with human physical interactomic data, we find that on average, proteins endowed with many interaction partners have disease related variations that are solvent exposed and are characterized by low free energy change values. Our results support the hypothesis that, besides protein stability perturbation, impairment of protein-protein interaction can be also a major mechanism explaining the relation between variations and diseases.

\section{Methods}

\section{Data set}

We downloaded from the Humsavar dataset (release 2015 10 of 14 Oct 2015) a collection of 27,185 variations related to 3082 OMIM diseases, on 2367 different human proteins and retained only proteins endowed with a PDB structure (3D) covering at least $70 \%$ of the protein sequence. The PDBSWS resource [20] (August 2015 update) was adopted to map the UniProt sequences onto the PDB structures. We ended up with a dataset of 4717 variations related to 484 . OMIM diseases on 368 proteins endowed with PDB structures with resolution lower 
than $3.0 \AA$ (OMIM set). On the same proteins, we also collected 685 polymorphism lacking evidence of association to disease (POLY set).

To train/test (by adopting a cross validation procedure) the predictors, we used S2648, a dataset that was originally derived from the ProTherm database [9] and corrected by the authors of the PoPMuSiC algorithm [11]. It comprises 2648 variations out of 132 different proteins endowed with a $3 \mathrm{D}$ structure. We also evaluated the predictor performances on a blind test of 351 variations in 60 proteins, and on 42 variations of the P53 protein not included in the training set and previously described in [12].

\section{INPS3D: a structure based method for the prediction of} free energy changes upon protein variations

Here we introduce INPS3D that exploits both sequence and structural information to predict the protein stability changes upon single point mutation. INPS3D takes advantage of the recently released INPS [15] that, starting only from protein sequence, performs similarly to the state-of the-art methods based on protein structure. INPS3D is based on nine input features based on protein sequence and structure. The features extracted from protein sequence are, [15]: 1) substitution score derived from the Blosum62 matrix; 2-3) Kyte-Doolittle hydrophobicity scores of native and mutated residues; 4) mutability index of the native residue; 5-6) molecular weights of native and mutated residues; 7) the difference in the alignment score between the native and mutated sequences and an HMM encoding evolutionary information of the target sequence. Two additional real-valued features derived from the protein structures are: 8) the solvent accessibility of the mutated residue, 9) the energy difference between native and mutated proteins. The solvent accessibility is computed with the DSSP method [21] and normalized as previously described [22]. The energy difference is evaluated by using the residue-based contact potential described in [23]. We consider that two residues are in contact if the minimal distance between all the atoms (not including hydrogen atoms) of two residues is $\leq 5 \AA$. We used the coordinates of the native protein to compute the contact energy and the energy difference as:

$$
\sum_{r} P(r, w)-P(r, m)
$$

where $P$ is the contact potential, $w$ is the wild-type residue, $m$ is the mutated residue, and the $r$-index runs over the list of $w$-neighbouring residues. We tested several other potentials, but the performances were similar or lower than those here reported. INPS3D is based on a Support Vector Regression model (SVR) trained on the same dataset adopted for INPS (see data set section). The adopted conventions on the sign are such as when predicting the $\Delta \Delta G$ associated to a variation, positive values refer to the protein stabilization and negative values to protein destabilization.

\section{Analysis of protein surfaces}

The solvent accessible surface area of residues in wildtype proteins has been evaluated with the DSSP program [21]. In order to obtain the Relative Solvent Accessibility (RSA), solvent accessibility areas were normalized to the residue-specific maximum solvent accessible area, as previously reported [22]. Residues with RSA $\geq 0.2$ are classified as accessible, residues with $\mathrm{RSA}<0.2$ are classified as buried. RSA has been measured on both the protein isolated chain and the protein complex, as downloaded from the repository of "biological assemblies" of the Protein Data Base [http://www.rcsb.org/ $\mathrm{pdb} /$ download/download.do\#Structures]. To define the interaction interface of the complex, we collected the set of residues that are solvent accessible in the isolated chain and are buried in the complex.

\section{Interactomics analysis}

Interacting partners of each protein were retrieved from the IntAct database [24] as downloaded from the IntAct FTP site as to November 2015. The search in the IntAct file was performed using the UniProtKB code and excluding the negative interaction data. The statistical analysis was performed considering only the proteins present in the dataset, at least in one entry.

Table 1 Performance of INPS3D and other state-of-the-art predictors

\begin{tabular}{llll}
\hline Method & $\begin{array}{l}\text { Cross-validation (2648 variations } \\
\text { on 132 proteins) }\end{array}$ & $\begin{array}{l}\text { Blind test set (351 variations } \\
\text { on 60 proteins) }\end{array}$ & $\begin{array}{l}\text { Blind test set (42 variations } \\
\text { on P53 protein) }\end{array}$ \\
\hline INPS $^{\mathrm{b}}$ & $0.53 / 1.29^{\mathrm{a}}$ & $0.68 / 1.26^{\mathrm{a}}$ & $0.71 / 1.49^{\mathrm{a}}$ \\
INPS3D $^{\text {a }}$ & $0.58 / 1.20^{\mathrm{a}}$ & $0.72 / 1.15^{\mathrm{a}}$ & $0.76 / 1.35^{\mathrm{a}}$ \\
MAESTRO $^{\mathrm{a}}$ & $0.63 / 1.17^{\mathrm{a}}$ & $0.71 / 1.16^{\mathrm{a}}$ & $0.44 / 1.71^{\mathrm{a}, \mathrm{e}}$ \\
mCSM $^{\mathrm{d}}$ & $0.51 / 1.26^{\mathrm{a}}$ & $0.67 / 1.19^{\mathrm{a}}$ & $0.68 / 1.40^{\mathrm{a}}$ \\
\hline
\end{tabular}

apearson's correlation coefficient/standard error ( $\mathrm{kcal} / \mathrm{mol})$

Data are from ${ }^{b}[15] ;{ }^{c}[13] ;{ }^{d}[12],{ }^{e}$ this work, respectively 


\section{Results and discussion INPS3D at work}

INPS3D is a new tool for predicting the change of protein folding free energy induced by single residue variations. The performance of the structure based predictor along with that of the sequence based one [15] are shown in Table 1. We report statistical scores obtained benchmarking the predictors with a more stringent per-protein cross-validation procedure [15] on the S2648 set previously described [11], and on a blind test set including some 351 variations in 60 proteins, and a P53 data set (both not included in the training set). Results, reported in Table 1, indicate that INPS3D outperforms INPS, exploiting structure based features not present in the INPS input encoding. INPS3D well compares with the performances obtained with structure-based stateof-the-art methods, mCSM [12], and MAESTRO, recently made available as web server [13].

\section{Predicting the effect of disease related, single residue variations on the stability of OMIM linked proteins} We applied INPS (sequence based), INPS3D (structure based) and MAESTRO (structure based) to the OMIM variation set for estimating the change in protein folding free energy induced by the disease-related variations. For sake of comparison we also ran the tools on the POLY set, containing variations not related to diseases, on the same OMIM proteins. We used polymorphisms from the very same proteins that have also variations related to diseases, in order to constrain the $\Delta G$ value of the folded form and avoid possible biases due to the inclusion of other proteins. The results (Fig. 1) confirm that disease related variations tend to produce a larger effect on protein stability than polymorphisms, which, on the other hand, appear to promote free energy perturbations mostly distributed within $+/-1 \mathrm{kcal} / \mathrm{mol}$. The result is confirmed by all the predictors. INPS3D predicts that $80 \%$ of polymorphisms and $56 \%$ of disease causing variations promote a $|\Delta \Delta \mathrm{G}| \leq 1 \mathrm{kcal} / \mathrm{mol}$ with respect to the corresponding native protein.

The results are similar with INPS; with Maestro, the fraction of disease-related variations predicted with low $|\Delta \Delta \mathrm{G}|$ values increases to $74 \%$ of the total. Our results, obtained with three independent predictors, corroborate the notion that protein stability perturbation (as detected from the predicted $|\Delta \Delta \mathrm{G}|>1 \mathrm{kcal} / \mathrm{mol}$ ) is associated to disease-related variations. However, at least half of the OMIM set is predicted to promote only a slight change in protein stability (within a range of about $1 \mathrm{kcal} / \mathrm{mol}$ in absolute value). The observation poses the question as to whether the thermodynamic property of the protein variant (albeit predicted) can be linked to some structural/ functional feature of the variation, specifically when it is disease causing. Many investigations addressed the issue of which structural features could be associated to disease related variations ([25-29] and references therein). Conclusions are that genetic variations can have dramatic effects on protein stability, hydrogen bonding networks, conformational dynamics, protein activity and protein interaction networks, particularly at the level of functional assemblies [28]. More recently the correlation between the probability of perturbing the protein stability and that of being disease causing was improved [19] with respect to previous data [16]. However, here our analysis addresses the issue from a different perspective: considering that we have predictors of protein stability, the problem is to which extent they label the overall protein in/stability in relation to the corresponding disease related mutation. We find that a high fraction of the protein variants carrying disease-related mutations are predicted with a low $|\Delta \Delta G|$ value, rather independently of the method (compare the INPS3D to MAESTRO results).

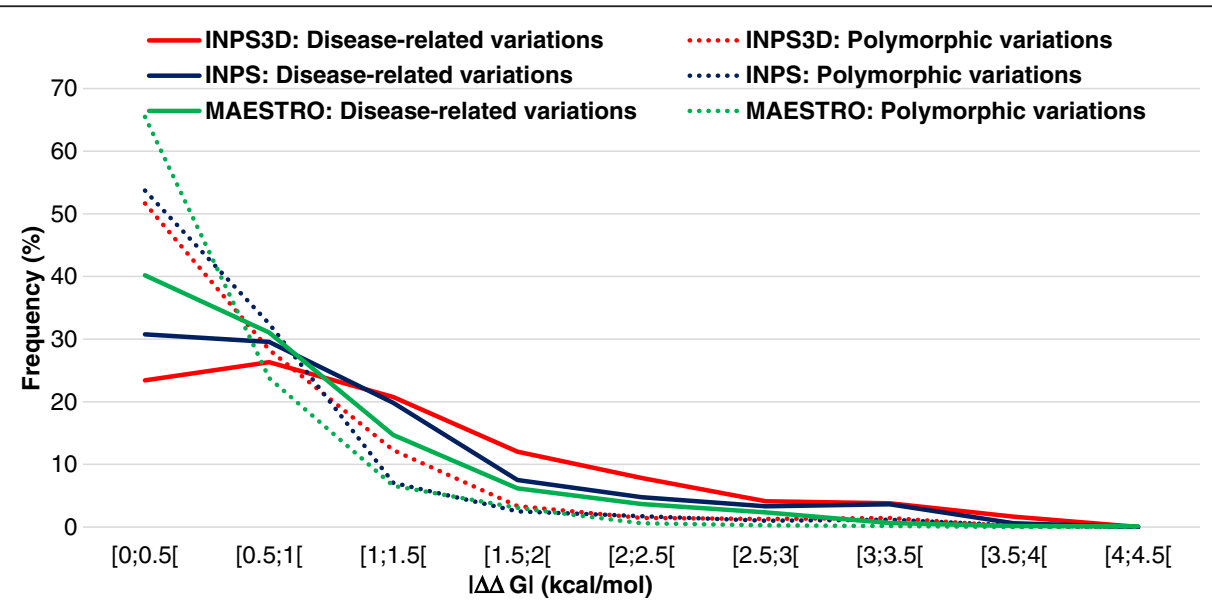

Fig. 1 Distribution of the absolute value of the $\triangle \triangle G$ predicted with INPS3D, MAESTRO and INPS. The set includes 4717 disease related variations and 687 polymorphisms in 368 OMIM proteins 


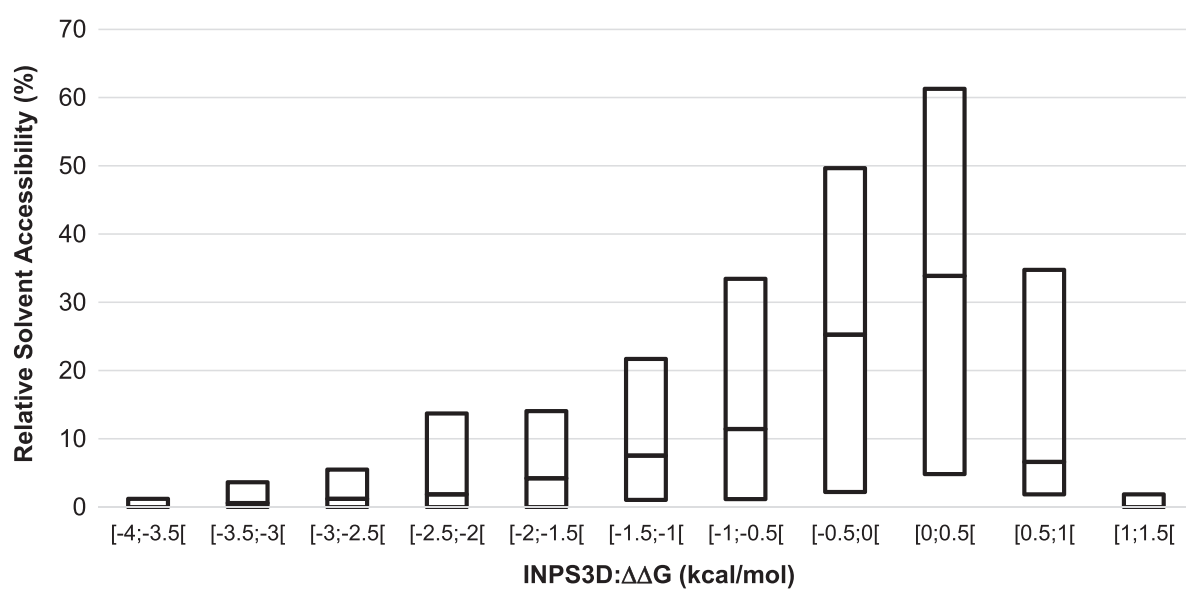

Fig. 2 Relative Solvent Accessibility of the variations as a function of $\Delta \Delta G$ predicted for the variants of the OMIM set. The box-plot reports the median and the lower and upper quartiles of the distribution of relative solvent accessibility for each interval of $\Delta \Delta \mathrm{G}$

\section{Protein $|\Delta \Delta \mathrm{G}|$ values and structural/functional properties} of the variations

In the following we will consider how some structural properties can be clustered considering perturbing and non- perturbing predicted $|\Delta \Delta G|$ values. The analysis focuses on the Relative Surface Accessibility (RSA), on the propensity of the variation to be or not in an interaction patch, and finally on the relation of the protein variant to be in physical interaction with other proteins, considering $\Delta \Delta \mathrm{G}$ values predicted with INPS3D.

We analyse the distribution of the relative solvent accessibility (RSA) of the disease related mutations as a function of the free energy change predicted for the corresponding protein variant. Boxplots in Fig. 2 show that the median and the upper quartile values of RSA are higher in the intervals with $\Delta \Delta G$ values close to zero. This indicates that disease related variations with low $\Delta \Delta G$ values have a more spread out distribution of RSA, and then a larger probability to be solvent accessible.
In Fig. 3, the distribution of the fraction of solvent accessible variations is plotted as a function of the $|\Delta \Delta G|$ values for disease related and polymorphic protein variants. Low $|\Delta \Delta \mathrm{G}|$ values are apparently common both to disease causing and polymorphic variations, when they are located in accessible protein sites.

A detailed grouping of the different behaviour of the structural properties of the OMIM related variations is shown in Tables 2 and 3, as a function of the thermodynamic property of the protein variant. Here we focus also on the difference among monomers and assemblies (as documented in the Protein Data Bank, http:// www.rcsb.org/pdb/download/download.do\#Structures), in order to highlight the role of protein-protein interactions, when present, in the biological functional unit. As an additional feature, we also included the likelihood of each variations to be or not in an interaction patch (computed with our PRED-PPI, [30]). It appears that disease related mutations in proteins variants with low $|\Delta \Delta \mathrm{G}|$ values, when solvent

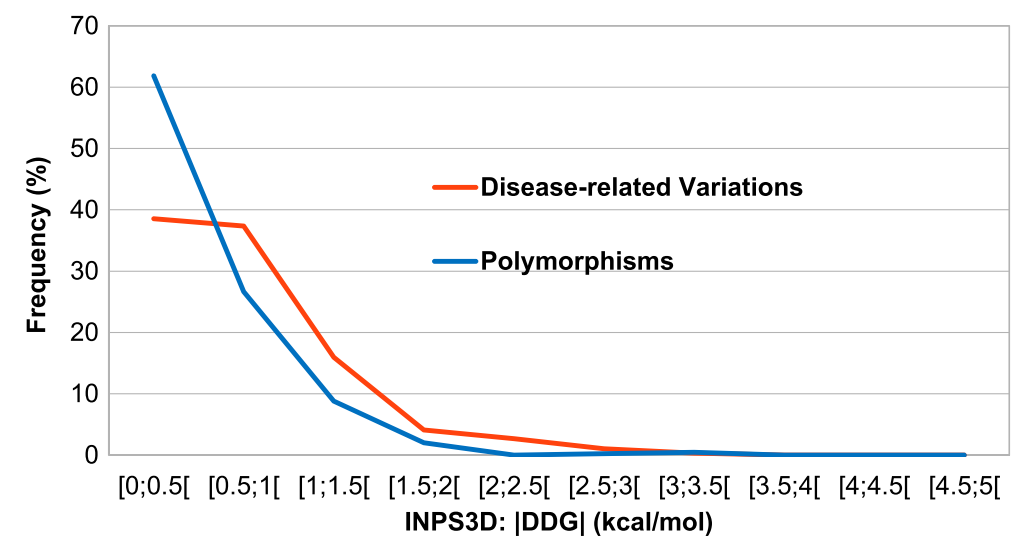

Fig. 3 Frequency of the solvent accessible variations as a function of $\Delta \Delta G$ predicted for the protein variants of the OMIM set 
Table 2 Relation between thermodynamic properties and structural properties in proteins with biologically functional monomeric assembly

\begin{tabular}{lll}
\hline Disease-related variant & RSA $\geq 0.20$ & RSA $<0.20$ \\
\hline$|\Delta \Delta G| \leq 1$ & $562(23.4 \%)^{\mathrm{a}} 398$ & $756(31.4 \%)^{\mathrm{a}} 39$ \\
$|\Delta \Delta \mathrm{G}|>1$ & $176(7.3 \%)^{\mathrm{a}} 120$ & $907(37.8 \%)^{\mathrm{a}} 36$ \\
Polymorphic variant & & \\
$|\Delta \Delta \mathrm{G}| \leq 1$ & $194(59.0 \%)^{\mathrm{a}} 110$ & $72(21.9 \%)^{\mathrm{a}} 3$ \\
$|\Delta \Delta \mathrm{G}|>1$ & $22(6.7 \%)^{\mathrm{a}} 10$ & $41(12.5 \%)^{\mathrm{a}} 0$
\end{tabular}

${ }^{a}$ Number of residue predicted to be part of a protein-protein interaction patch (for details on the prediction method, see [30]). Predicted set: 2401 disease related variations and 329 polymorphic variations in 177 proteins

exposed (RSA $\geq 0.20$ ), have also a tendency to be in interaction sites. The property is shared, as expected, with variations that highly perturb protein stability and with polymorphic ones. The low accessibility, in all cases, well agrees with a propensity of being in interaction sites ranging from 0 to $5 \%$. The value can be considered indicative of the possible range of the false positive rate of the predictor, trained and tested on accessible interaction sites and for which the OMIM set of disease related and polymorphic variations is a blind test set.

Distinguishing functional monomeric from multimeric biological assemblies highlights the relevance of the variations when they are located at the interface of protein complexes [28]. In Table 3, the same grouping of Table 2 is therefore shown for proteins with a biologically functional assembly, as documented in the PDB. Here, it appears that only a small fractions of the total number of disease related mutations in the set occurs at the monomer interface (compare Monomer and Complex at RSA $\geq$ 0.20 ) and concomitantly also the number of interaction sites predicted on the complex interface is very low.

From the data reported in Tables 2 and 3, it can be computed that about $70 \%$ of the disease causing variations with high accessible surface in monomers are predicted to be part of an interaction patch. The result is particularly significant considering that the fraction of all accessible residues predicted in interaction patches on the same 368 proteins is $55 \%$.

Summing up, we show that disease related variations in proteins can promote a low $|\Delta \Delta \mathrm{G}|$ value, particularly when they are located in accessible sites that are also interacting sites.

As a follow up, one may consider to which extent protein variants with disease-related mutations located in solvent exposed sites and slightly perturbing the stability, are or not involved in interaction networks of physical interaction, as available in IntAct [24]. We collected from IntAct the number of interacting partners for each protein and analysed it as a function of the fraction of solvent accessible, non-perturbing variations (Fig. 4). The upper quartile and the mean values of the number of interacting partners per protein increase as the fraction of disease related variations predicted as nonperturbing increases. When all the solvent exposed disease related mutations (RSA $\geq 20 \%$ ) per protein are related to the number of the corresponding protein interacting partners (Fig. 5), the trend is different from that observed in Fig. 4. This observation highlights the role of predicted $\Delta \Delta G$ values for determining the relation among protein variants with disease-related mutations located in solvent exposed sites and slightly perturbing the stability, and the number of interacting partners in a protein-protein interaction network.

The proteins endowed with a large amount of nonperturbing and solvent exposed disease related variations seem to play a central role in the human protein-protein interaction network. Likely, a variation on the protein surface can affect the interaction affinity, affecting important biological pathways and leading to an altered phenotype, as recently described [31]. Out of the 43

Table 3 Relation between thermodynamic properties and structural properties in proteins with biologically functional multimeric assembly

\begin{tabular}{lll}
\hline Disease-related variations & RSA $\geq 0.20$ & RSA $<0.20$ \\
\hline$|\Delta \Delta G| \leq 1$ & $660(28.5 \%)$ Monomer $^{\mathrm{a}} 465$ & $650(28.0 \%)$ Monomer 24 \\
& $550(25.0 \%)$ Complex $^{\mathrm{a}} 421$ & $760(31.5 \%)$ Complex 68 \\
$|\Delta \Delta \mathrm{G}|>1$ & $213(9.2 \%)$ Monomer $^{\mathrm{a}} 152$ & $793(34.2 \%)$ Monomer 24 \\
& $196(8.5 \%)$ Complex $^{\mathrm{a}} 140$ & $810(35.0 \%)$ Complex 36 \\
Polymorphic variations & & $84(23.6 \%)$ Monomer 5 \\
$|\Delta \Delta \mathrm{G}| \leq 1$ & $198(55.6 \%)$ Monomer $^{\mathrm{a}} 131$ & $96(27.0 \%)$ Complex 17 \\
& $186(52.2 \%)$ Complex $^{\mathrm{a}} 119$ & $45(12.6 \%)$ Monomer $^{\mathrm{a}} 9$ \\
$|\Delta \Delta \mathrm{G}|>1$ & $29(8.1 \%)$ Monomer $^{\mathrm{a}} 21$ & $45(12.6 \%)$ Complex 9
\end{tabular}

${ }^{a}$ Number of residue predicted to be part of a protein-protein interaction patch. 2316 disease related variations and 356 polymorphic variations in 191 proteins Predictions of INPS-3D and PRED-PPI are independent of the assembly state. RSA values were independently estimated on the monomeric and the complex structures 


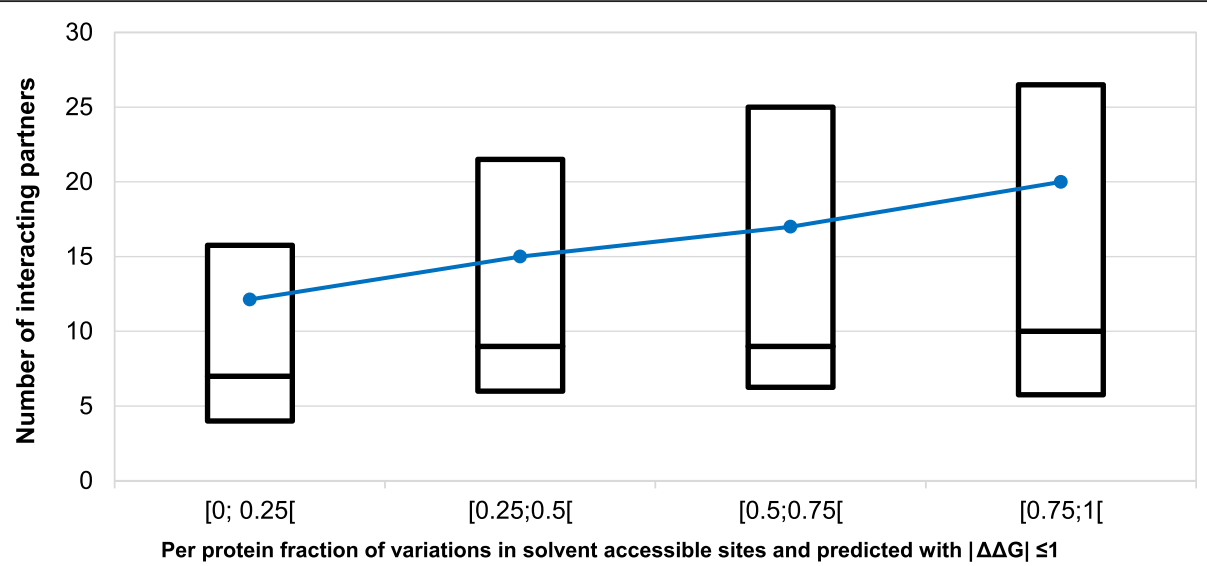

Fig. 4 Relation between the per-protein fraction of non-perturbing, solvent accessible variations and the corresponding number of the wild-type partners of interactions in the human interactome. The box-plot reports the median and the lower and upper quartiles of the number of interactions present in IntAct as a function of the fraction of solvent accessible, non-perturbing variations. The dashed blue line connects the average values. Non perturbing variations are those predicted to promote a $|\Delta \Delta \mathrm{G}| \leq 1 \mathrm{kcal} / \mathrm{mol}$ with INPS3D and found in protein sites that are solvent accessible. Data refers to 170 proteins with 4037 variations of our data set. Proteins with less than 5 disease-related variations or without interactomic data reported in IntAct are excluded

proteins for which at least $50 \%$ of disease related variations are solvent exposed and predicted with $|\Delta \Delta \mathrm{G}| \leq 1$, $42 \%$ are involved in differentiation and development processes (including insulin, calmodulin, noggin, angiogenin), $40 \%$ are involved in signalling processes (including the GTPases KRAS, HRAS and NRAS, the serine/threonine kinases PIK3CA and CHEK2), $23 \%$ are structural and adhesion proteins (e.g., actins ACTA1, ACTG2, tubulin TUBA1A and integrin $\beta 2$ ).

\section{Conclusions}

We address the problem of the perturbations of the protein stability by disease causing variations on a set of
OMIM related proteins whose native structure is well solved. To this aim we implemented INPS3D, a tool for computationally estimating the change in $\Delta \Delta G$ value associated to single residue variations, taking as input protein structure. Our strategy is to adopt a predictor that scores at the state-of-the-art and we compare its performance to other state-of-the-art predictors. INPS3D exploits information extracted from protein structures and outperforms the recently released INPS, based only on sequence information. Moreover INPS3D outperforms state-of-the-art structure-based methods that perform similarly to INPS and well compares with MAESTRO, which recently became available as a web

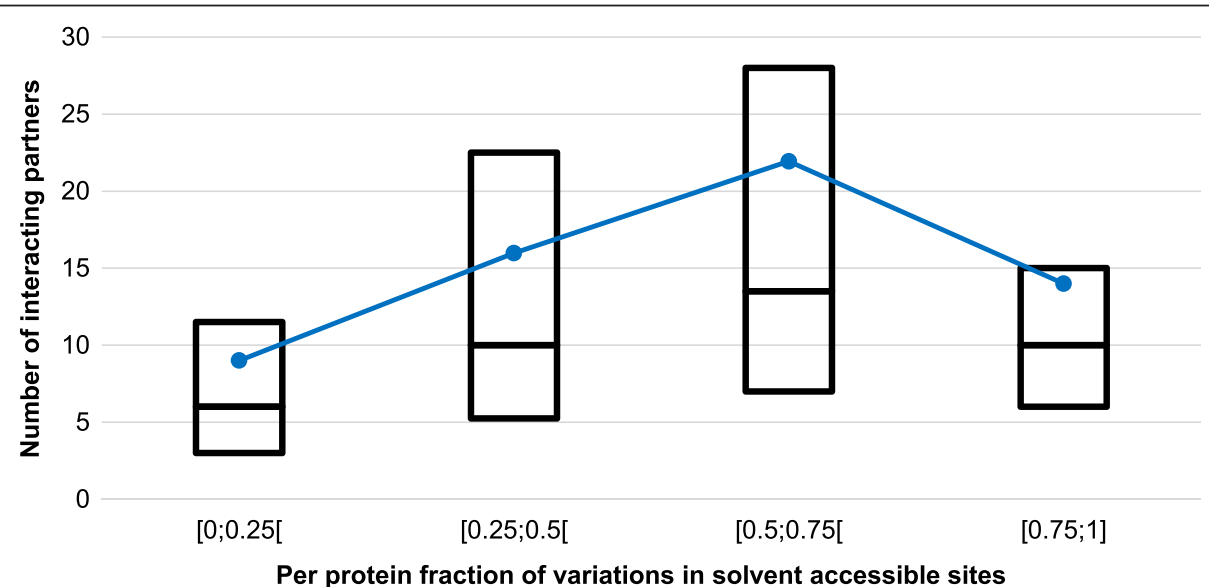

Fig. 5 Relation between the per-protein fraction of solvent accessible variations and the corresponding number of the wild-type partners of interactions in the human interactome. The box-plot reports the median and the lower and upper quartiles of the number of interactions present in IntAct as a function of the fraction of solvent accessible variations. The dashed blue line connects the average values. Data refers to 170 proteins with 4037 variations of our data set. Proteins with less than 5 disease-related variations or without interactomic data reported in IntAct are excluded 
server [13]. Both predictors agree up to $90 \%$ even in regions of $|\Delta \Delta G|$ values that can be considered below the error limit of the predictors. We found that OMIM disease-related variations in proteins generally promote a much larger effect on protein stability than polymorphisms non-associated to diseases on the same proteins, confirming that stability perturbation plays a crucial role in impairing protein function (recently confirmed also in [31]). Nevertheless, a significant fraction of disease related variations is predicted to have a small perturbation effect on protein stability: about $50 \%$ of variations promote a $|\Delta \Delta \mathrm{G}|<1 \mathrm{kcal} / \mathrm{mol}$. The structural analysis of the corresponding proteins reveals that disease-related variations with a slight effect on protein stability often occur on the protein surface suggesting that they can affect the interaction of the proteins within biological functional networks. The analysis of protein-protein interaction networks corroborates the hypothesis that proteins with many non-perturbing disease-related variations are more connected in the human physical interactome (IntAct) than proteins with variations predicted with $|\Delta \Delta \mathrm{G}|>1 \mathrm{kcal} / \mathrm{mol}$. The results are however indicative. The error associated to the computed $|\Delta \Delta \mathrm{G}|$ value by our predictors (Table 1 ) is competing with the range of small changes in protein stability and this could increase the number of variations actually destabilising protein stability. It should also be mentioned that for each protein other features that are not exploited in this analysis (e.g., solubility, post-translational modifications, subcellular location, level of expression, etc.) may be considered when labelling a variations as disease causing.

\section{Abbreviations}

RSA: relative solvent accessibility.

\section{Funding}

PRIN 2010-2011 project 20108XYHJS (to P.L.M.) (Italian MIUR); COST BMBS Action TD1101 and Action BM1405 (European Union RTD Framework Program, to R.C); PON projects PON01_02249 and PAN Lab PONa3_00166 (Italian Miur to R.C. and P.L.M.); FARB UNIBO 2012 (to R.C.).

\section{Availability of data and material}

The method is available at http://inpsmd.biocomp.unibo.it/inpsSuite/default/ index3D. Data are available upon request.

\section{Authors' contributions}

PLM, PF, CS and RC conceived and designed the work and wrote the paper. PF and CS implemented and tested INPS3D. PLM and RC analysed and interpreted data on disease related variations. GB and FA curated the datasets and collaborated in data analysis. All authors critically revised and approved the manuscript.

\section{Competing interests}

The authors declare that they have no competing interests.

\section{Consent for publication}

Not applicable.

Ethics approval and consent to participate Not applicable.

\section{Declarations}

The publication charges for this article were funded by PRIN 2010-2011 project (Italian Ministry of Education, University and Research, cod 20108XYHJS). This article has been published as part of BMC Genomics Volume 17 Supplement 2, 2016: Proceedings of Varl-SIG 2015: Identification and annotation of genetic variants in the context of structure, function, and disease. The full contents of the supplement are available online at https://bmcgenomics.biomedcentral.com/articles/ supplements/volume-17-supplement-2.

\section{Author details}

${ }^{1}$ Biocomputing Group, University of Bologna, Via San Giacomo 9/2, 40126 Bologna, Italy. ${ }^{2}$ Department BiGeA, University of Bologna, Via Selmi 3, 40126 Bologna, Italy. ${ }^{3}$ Department BCA, University of Padova, Viale Università 16, 35020 Legnaro (PD), Italy.

Published: 23 June 2016

\section{References}

1. Lu YF, Goldstein DB, Angrist M, Cavalleri G. Personalized medicine and human genetic diversity. Cold Spring Harb Perspect Med. 2014;4:a008581.

2. Ashley EA. The precision medicine initiative: a new national effort. JAMA. 2015;313:2119-20.

3. Brookes AJ, Robinson PN. Human genotype-phenotype databases: aims, challenges and opportunities. Nat Rev Genet. 2015;16:702-15.

4. Li B, Krishnan VG, Mort ME, Xin F, Kamati KK, Cooper DN, Mooney SD, Radivojac P. Automated inference of molecular mechanisms of disease from amino acid substitutions. Bioinformatics. 2009;25:2744-50.

5. Calabrese R, Capriotti E, Fariselli P, Martelli PL, Casadio R. Functional annotations improve the predictive score of human disease-related mutations in proteins. Hum Mutat. 2009:30:1237-44.

6. Adzhubei IA, Schmidt S, Peshkin L, Ramensky VE, Gerasimova A, Bork P, Kondrashov AS, Sunyaev SR. A method and server for predicting damaging missense mutations. Nat Methods. 2010;7:248-9.

7. Thusberg J, Olatubosun A, Vihinen M. Performance of mutation pathogenicity prediction methods on missense variants. Hum Mutat. 2011; 32:358-68.

8. Bromberg Y, Rost B. SNAP: predict effect of non-synonymous polymorphisms on function. Nucleic Acids Res. 2007;35:3823-35.

9. Kumar MD, Bava KA, Gromiha MM, Prabakaran P, Kitajima K, Uedaira H, Sarai A. Protherm and Pronit: thermodynamic databases for proteins and protein-nucleic acid interactions. Nucleic Acids Res. 2006;34:D204-6.

10. Capriotti E, Fariselli P, Rossi I, Casadio R. A three-state prediction of single point mutations on protein stability changes. BMC Bioinformatics. 2008;9 Suppl 2:S6.

11. Dehouck Y, Kwasigroch JM, Gilis D, Rooman M. PoPMuSiC 2.1: a web server for the estimation of protein stability changes upon mutation and sequence optimality. BMC Bioinformatics. 2011;12:151.

12. Pires DE, Ascher DB, Blundell TL. mCSM: predicting the effects of mutations in proteins using graph-based signatures. Bioinformatics. 2014;30:335-42

13. Laimer J, Hiebl-Flach J, Lengauer D, Lackner P. MAESTROweb: a web server for structure based protein stability prediction. Bioinformatics. 2016. [Epub ahead of print].

14. Khan S, Vihinen M. Performance of protein stability predictors. Hum Mutat. 2010;31:675-84.

15. Fariselli P, Martelli PL, Savojardo C, Casadio R. INPS: predicting the impact of non-synonymous variations on protein stability from sequence. Bioinformatics. 2015;31:2816-21.

16. Casadio R, Vassura M, Tiwari S, Fariselli P, Martelli PL. Correlating diseaserelated mutations to their effect on protein stability: a large-scale analysis of the human proteome. Hum Mutat. 2011;32:1161-70.

17. Petukh M, Kucukkal TG, Alexov E. On human disease-causing amino acid variants: statistical study of sequence and structural patterns. Hum Mutat. 2015;36:524-34.

18. Pal LR, Moult J. Genetic basis of common human disease: insight into the role of missense SNPs from genome-wide association studies. J Mol Biol. 2015:427:2271-89.

19. Peng $Y$, Alexov E. Investigating the linkage between disease-causing amino acid variants and their effect on protein stability and binding. Proteins. 2016;84:232-9. 
20. Martin AC. Mapping PDB, chains to UniProtKB entries. Bioinformatics. 2005 21:4297-301.

21. Kabsch W, Sander C. Dictionary of protein secondary structure: pattern recognition of hydrogen-bonded and geometrical features. Biopolymers. 1983;22:2577-637.

22. Rost B, Sander C. Conservation and prediction of solvent accessibility in protein families. Proteins. 1994;20:216-26.

23. Bastolla U, Farwer J, Knapp EW, Vendruscolo M. How to guarantee optimal stability for most representative structures in the protein data bank. Proteins. 2001;44:79-96.

24. Kerrien S, Aranda B, Breuza L, Bridge A, Broackes-Carter F, Chen C, Duesbury M, Dumousseau M, Feuermann M, Hinz U, Jandrasits C, Jimenez RC, Khadake J, Mahadevan U, Masson P, Pedruzzi I, Pfeiffenberger E, Porras P, Raghunath A, Roechert B, Orchard S, Hermjakob H. The IntAct molecular interaction database in 2012. Nucleic Acids Res. 2012;40(Database issue): D841-6.

25. Tokuriki N, Stricher F, Schymkowitz J, Serrano L, Tawfik DS. The stability effects of protein mutations appear to be universally distributed. J Mol Biol. 2007;369:1318-32

26. Gong S, Blundell TL. Structural and functional restraints on the occurrence of single amino acid variations in human proteins. PLoS One. 2010;5:e9186.

27. David A, Razali R, Wass MN, Sternberg MJ. Protein-protein interaction sites are hot spots for disease-associated nonsynonymous SNPs. Hum Mutat. 2012;33:359-63.

28. Wei $\mathrm{Q}, \mathrm{Xu} \mathrm{Q}$, Dunbrack Jr RL. Prediction of phenotypes of missense mutations in human proteins from biological assemblies. Proteins. 2013:81:199-213.

29. Stefl S, Nishi H, Petukh M, Panchenko AR, Alexov E. Molecular mechanisms of disease-causing missense mutations. J Mol Biol. 2013;425:3919-36.

30. Bartoli L, Martelli PL, Rossi I, Fariselli P, Casadio R. The prediction of protein-protein interacting sites in genome-wide protein interaction networks: the test case of the human cell cycle. Curr Protein Pept Sci. 2010;11:601-8.

31. Wang X, Wei X, Thijssen B, Das J, Lipkin SM, Yu H. Three-dimensional reconstruction of protein networks provides insight into human genetic disease. Nat Biotechnol. 2012;30:159-64.

\section{Submit your next manuscript to BioMed Central and we will help you at every step:}

- We accept pre-submission inquiries

- Our selector tool helps you to find the most relevant journal

- We provide round the clock customer support

- Convenient online submission

- Thorough peer review

- Inclusion in PubMed and all major indexing services

- Maximum visibility for your research

Submit your manuscript at www.biomedcentral.com/submit

C Biomed Central 